\title{
Solution of the Stefan Problem: Silver Electrodeposition under Mass Transfer Control. The Transition from Diffusion to Advection Regime
}

\author{
M. A. Pasquale, S. L. Marchiano, J. L. Vicente, and A. J. Arvia* \\ Instituto de Investigaciones Físicoquimicas Teóricas y Aplicadas (INIFTA), \\ Universidad Nacional de La Plata-Consejo Nacional de Investigaciones Científicas y Técnicas, \\ Sucursal 4, Casilla de Correo 16, (1900) La Plata, Argentina
}

Received: May 6, 2004; In Final Form: June 15, 2004

\begin{abstract}
An analytical solution of Fick's diffusion equation for the formation of a 2D solid-phase involving a growing front (Stefan problem) position changing at a constant linear velocity is proposed. This solution comprises a first diffusion term that includes an exponential correction factor, and a second constant advection term that depends on the front velocity, and it predicts a kinetic transition from a diffusion to an advection-dominated mass transfer control in going from $t \rightarrow 0$ to $t \rightarrow \infty$. The validity of the analytical solution is tested using potentiostatic cathodic current transient data for silver electrodeposition in a quasi-2D rectangular cell with an initially plane plate cathode. Theoretical and experimental data fulfill a dimensionless correlation after experimental data are properly corrected for the effective cathode area.
\end{abstract}

\section{Introduction}

Convective diffusion differential equations have been solved for a number of cases in which the coordinates of the interfacial front remain constant. ${ }^{1,2}$ This situation, however, does not apply to solid/fluid interfaces in which a moving front is involved. ${ }^{3-6}$ Situations of this type occur in a diversity of converging phenomena in the areas of physics, chemistry, biology, and related subjects, in which the solution of those differential equations depends on the moving boundary conditions.

Mathematical solutions of moving boundary problems for heat transfer have been studied since about a century ago, ${ }^{7-13}$ a typical example being the displacement of icebergs. In contrast, scarce attention has been paid to the relevance of moving boundary problems in mass transport phenomena. ${ }^{14-16}$ These problems are often called Stefan problems, irrespective of the driven force. ${ }^{12}$

In general, diffusion equations can be solved by considering (i) a fixed boundary, (ii) a moving boundary, and (iii) a free boundary of either an explicit or an implicit type. Case (i) is found for mass transfer phenomena between different phases in which a steady boundary is involved, and then equations for each phase at their fixed spatial domain are considered. Conversely, the spatial domains are time-dependent in cases (ii) and (iii). For case (ii) the moving front fulfills a displacement law that is known a priori. In contrast, for case (iii) the boundary velocity equations of spatial domains are unknown and, therefore, new boundary conditions for solving the problem have to be found. These conditions would depend on the physical description of each problem. The explicit situation, in contrast to the implicit one, implies that the front velocity equation is available.

The Stefan problem appears for a process involving the growth of a solid material with a moving boundary under diffusion control. Such a process results in either the growth of the solid phase when impinging particles from a fluid phase are incorporated into the phase, or its disappearance when

* e-mail address: ajarvia@inifta.unlp.edu.ar; fax 54-221-425-4642. detaching particles move outward into the fluid phase. For the simplest one-dimensional description, the front displacement occurs perpendicularly with respect to the initial surface (substrate), that is, either negatively for a growing front or positively for a dissolving front. ${ }^{3,11}$ In this description it is assumed, as a first approach, that changes in the macroscopic roughness of the moving boundary and convective effects from density gradients can be disregarded.

This paper reports a solution of the Stefan problem at a moving boundary of a growing metal deposit under mass transport rate control. The proposed solution is reasonably fulfilled by data resulting from silver electrodeposition from a conventional plating solution using a quasi-2D-electrochemical cell. ${ }^{17-18}$

\section{Theoretical Approach}

2.1. Mathematical Solution. A 1D ( $y$-direction) diffusion process of species i perpendicular to a plane plate of infinite dimensions is expressed by Fick's equation

$$
D_{\mathrm{i}} \frac{\partial^{2} c_{\mathrm{i}}}{\partial y^{2}}=\frac{\partial c_{\mathrm{i}}}{\partial t}
$$

where $D_{\mathrm{i}}$ and $c_{\mathrm{i}}$ are the diffusion coefficient and the concentration of species $\mathrm{i}$ in the fluid phase, and $t$ is the time counted from the initiation of the process. The advance direction of the solid-phase front ( $y$-direction) is perpendicular to the plane plate. Equation 1 is solved with the following boundary conditions,

$$
\begin{gathered}
c_{\mathrm{i}}(y, 0)=c_{\mathrm{i}}^{0} \quad y \leq 0 \quad s(t)=s(0) \\
c_{\mathrm{i}}[s(t), t]=0 \quad t>0 \\
s(t)=\beta \cdot t \\
c_{\mathrm{i}}(y, t)=c_{\mathrm{i}}^{0} \quad y \rightarrow \infty
\end{gathered}
$$

$s(t)$ being the front displacement coordinate at time $t$, and $\beta$ the advance velocity of the moving front. Condition (2a) corre- 
sponds to the initially uniform reactant concentration in the plating solution. The initial silver growth front surface is equal to that of the inert substrate $(y=0)$ upon which the growth of the silver phase commences $(t=0)$. Condition (2b) indicates that the silver ion concentration just in front of the growing front is null for $t>0$, as expected from the mass transfer control of the electrochemical process. Condition (2c), usually called the Stefan condition, indicates the position of the silver front. Solving eq 1 with boundary conditions $(2 a-d)$, the explicit expression for $c_{\mathrm{i}}(y, t)$ results in

$$
\begin{array}{r}
c_{\mathrm{i}}=\frac{c_{\mathrm{i}}^{0}}{2}\left\{\exp \left[\left(\frac{\beta}{D_{\mathrm{i}}}\right) \cdot(\beta t-y)\right]\left[\operatorname{erf}\left(\frac{(y-\beta t)}{2 \sqrt{D_{\mathrm{i}} t}}\right)-1\right]+\right. \\
\left.\operatorname{erf}\left[\frac{y}{2 \sqrt{D_{\mathrm{i}}}}\right]+1\right\}
\end{array}
$$

Equation 3 fulfills boundary conditions (2a) and (2b).

Let us consider the electrodeposition of a metal $i$ from a silver plating solution on a plane plate silver substrate under mass transport control. For metal electrodeposition under diffusion control the instantaneous rate equation given in terms of the cathodic current density $j_{\mathrm{c}}(t)$ is

$$
j_{\mathrm{c}}(t)=z_{\mathrm{i}} \mathrm{F} D_{\mathrm{i}}\left[\frac{\partial c_{\mathrm{i}}(y, t)}{\partial y}\right]_{y=\beta t}
$$

$z_{\mathrm{i}}, c_{\mathrm{i}}$, and $\mathrm{F}$ being the electric charge per reacting silver ion $\left(z_{i}=1\right)$, the silver ion concentration in the plating solution, and the Faraday constant, respectively. Then,

$$
\begin{aligned}
& j_{\mathrm{c}}(t)=z_{\mathrm{i}} \mathrm{F} c_{\mathrm{i}}^{0}\left\{\left(\frac{D_{\mathrm{i}}}{\pi t}\right)^{1 / 2} \exp \left[\left(-\frac{\beta^{2} t}{4 D_{\mathrm{i}}}\right)\right]+\right. \\
&\left.\frac{\beta}{2}\left[\operatorname{erf}\left[\frac{\beta}{2}\left(\frac{t}{D_{i}}\right)^{1 / 2}\right]+1\right]\right\}
\end{aligned}
$$

The first right-hand-side (rhs) term in eq 5 is the product of the diffusion term for a fixed plane plate, and the exponential factor that produces a decrease in $j_{\mathrm{c}}(t)$ faster than that predicted by the solution of Fick's equation for fixed boundary, whereas the second rhs term increases $j_{\mathrm{c}}(t)$ even for $t=0$. In eq 5 , the relative contribution of the first term becomes more remarkable for $t \rightarrow 0$. The limit of eq 5 for $t \rightarrow 0$ is

$$
\left[j_{\mathrm{c}}(t)\right]_{t \ll 1}=z_{\mathrm{i}} \mathrm{F} c_{\mathrm{i}}^{0}\left[\sqrt{\frac{D_{\mathrm{i}}}{\pi t}}+\frac{\beta}{2}\right]=z_{\mathrm{i}} \mathrm{F} c_{\mathrm{i}}^{0} \sqrt{\frac{D_{\mathrm{i}}}{\pi t}}+\frac{\left(j_{\mathrm{c}}\right)_{\mathrm{adv}}}{2}
$$

The second term for $t \rightarrow \infty$ leads to a steady regime that corresponds to advection control. The limit of eq 5 for $t \rightarrow \infty$ is

$$
\lim \left[j_{\mathrm{c}}(t)\right]_{t \rightarrow \infty}=z_{\mathrm{i}} \mathrm{F} c_{\mathrm{i}}^{0} \beta=\left(j_{\mathrm{c}}\right)_{\mathrm{adv}}
$$

Equation 7 corresponds to the advection limit to the mass transport process.

Accordingly, eq 5 predicts a transition in the mass-transfer electrodeposition process from a nonsteady to a steady regime.

To further explore the behavior of eq 5 , let us define the dimensionless current ratio $\rho(\tau)$

$$
\rho(\tau)=j_{\mathrm{c}}(\tau) /\left(j_{\mathrm{c}}\right)_{\mathrm{adv}}
$$

The dimensionless time $(\tau)$ is expressed by

$$
\tau=\beta^{2} t / 4 D_{\mathrm{i}}
$$

TABLE 1: Values of $\rho(\tau)$ and $\tau$ Calculated from Eq 9

\begin{tabular}{lccl}
\hline \multicolumn{1}{c}{$\rho(\tau)$} & $\tau$ & $\rho(\tau)$ & \multicolumn{1}{c}{$\tau$} \\
\hline 0 & - & 1.81818 & 1.100569 \\
0.10101 & 1.47577 & 1.91919 & 1.00483 \\
0.20202 & 1.25031 & 2.02020 & 1.00411 \\
0.30303 & 1.16035 & 2.12121 & 1.00351 \\
0.40404 & 1.11194 & 2.22222 & 1.003 \\
0.50505 & 1.08211 & 2.32323 & 1.00257 \\
0.60606 & 1.06221 & 2.42424 & 1.00221 \\
0.70707 & 1.04823 & 2.52525 & 1.0019 \\
0.80808 & 1.03806 & 2.62626 & 1.00164 \\
0.90909 & 1.03044 & 2.72727 & 1.00141 \\
1.01010 & 1.02461 & 2.82828 & 1.00122 \\
1.11111 & 1.02008 & 2.92929 & 1.00106 \\
1.21212 & 1.01651 & 3.03030 & 1.00092 \\
1.31313 & 1.01366 & 3.13131 & 1.00079 \\
1.41414 & 1.01137 & 3.23232 & 1.00069 \\
1.51515 & 1.00951 & 3.33333 & 1.0006 \\
1.61616 & 1.00798 & 3.43434 & 1.00052 \\
1.71717 & 1.00673 & 3.53535 & 1.00046
\end{tabular}

Then, by placing eq 5 and 7 into eq 8 , after considering the dimensionless time $\tau$ from eq 9 , the result is

$$
\rho(\tau)=\frac{1}{2}\left[\frac{1}{\sqrt{(\pi \tau)}} \exp (-\tau)+\operatorname{erf}(\sqrt{\tau})+1\right]
$$

Obviously, eq 10 for $\tau \rightarrow \infty$ leads to $\rho(\tau) \rightarrow 1$. Values of $\rho(\tau)$ resulting from eq 10 for different $\tau$ are assembled in Table 1 .

2.2. Results from the Mathematical Solution. Equation 5 predicts a complex behavior of potentiostatic current transients (Figure 1a) because the contributions of the diffusion and advection terms both depend on the value of $\beta$. The diffusion term in eq 5 decays faster than that predicted for pure diffusion, the faster its decay the greater the value of $\beta$ (Figure 1a). Correspondingly, the greater the value of $\beta$ the shorter the values of $t$ required for the advection regime. Likewise, for a constant value of $\beta$, the longer the value of $t$ the sooner the advection regime is attained. In this case, a linear $j_{\mathrm{c}}$ versus $\beta$ dependence is approached (Figure 1b) and the extent of the advection range increases with $\beta$.

The linear dependence of $j_{\mathrm{c}}$ on $\beta$ from eq 7 contrasts with the $\langle\beta\rangle^{1 / 2}$ dependence that would result, in general, from laminar flow $^{1}$ and from forced convection due to flow impinging perpendicularly on a plane plate electrode. ${ }^{19,20}$ The comparison of these behaviors (Figure 1b) establishes a clear distinction between the different hydrodynamic mechanisms.

\section{Experimental Approach}

3.1. Silver Electrocrystallization. The overall silver electrocrystallization process that takes place in aqueous solutions can be represented by the following reaction

$$
\begin{aligned}
& \left.\operatorname{Ag}^{+}(\mathrm{aq})+\mathrm{e}^{-} \text {(cathode }\right)= \\
& \left.\operatorname{Ag}^{+}(\text {crystal })+\mathrm{e}^{-} \text {(conduction band }\right)
\end{aligned}
$$

For reaction 11 , the exchange current density $j_{0}$ is strongly dependent on the distribution of crystallographic faces of the substrate, and on the density number of surface defects. ${ }^{21-24}$ For stepped polycrystalline silver in aqueous $1 \mathrm{M}$ silver sulfate at $298 \mathrm{~K}$, a value for $j_{0}$ of $24 \pm 5 \mathrm{~A} / \mathrm{cm}^{2}$ has been reported. ${ }^{21}$ The estimated value of $j_{0}$ for aqueous $2.4 \times 10^{-2} \mathrm{M}$ silver sulfate at $298 \mathrm{~K}$ is $0.57 \mathrm{~A} / \mathrm{cm}^{2}$. Accordingly, reaction 11 on this type of surface can be considered as one of the fastest, most reversible electrochemical processes. This explains why the rate of reaction 11 becomes diffusion controlled even at very low cathodic overpotentials. 

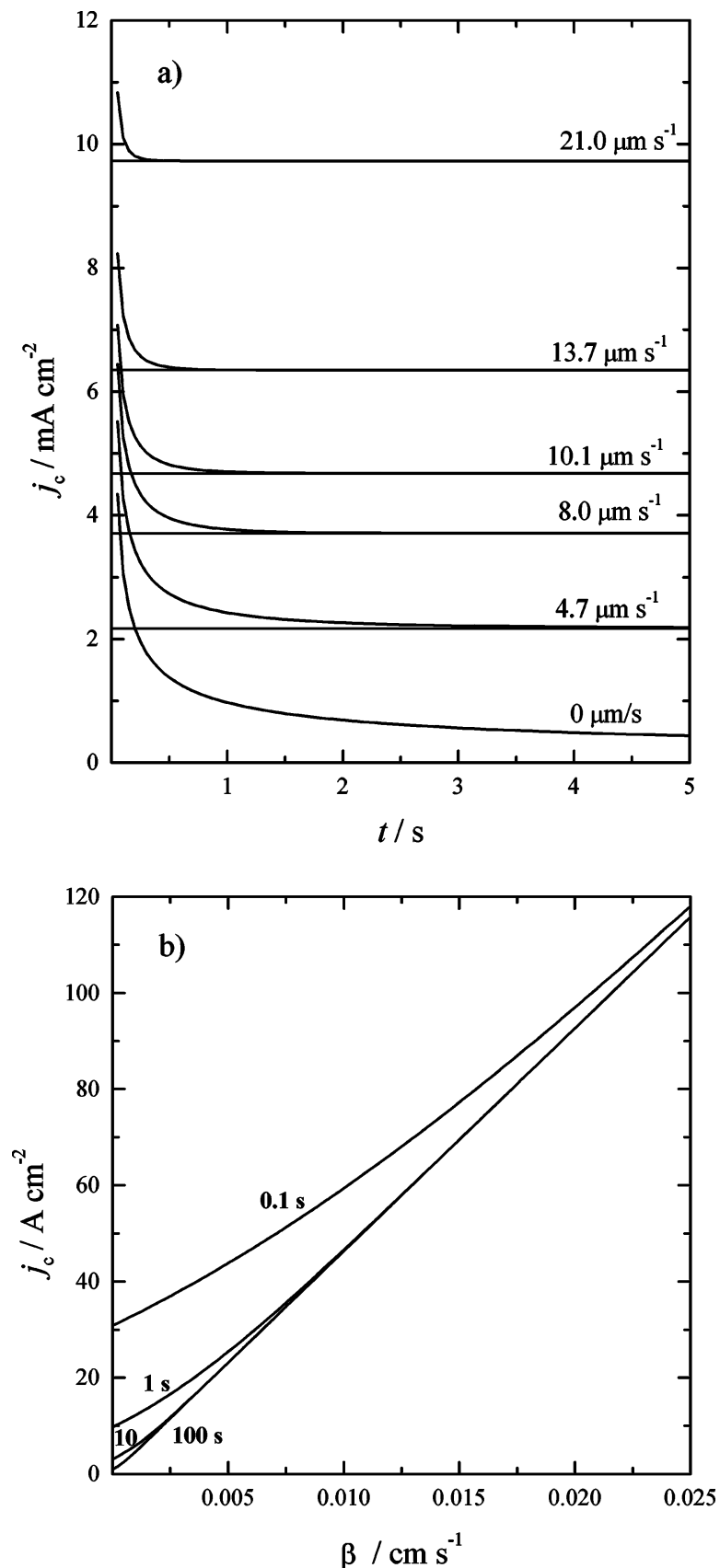

Figure 1. (a) Plots of eq 5 for different values of $\beta$, which are indicated in the figure. The height of the advection plateau is directly proportional to $\beta$. (b) $j_{\mathrm{c}}$ versus $\beta$ plots for different times (values of time are indicated in the plots).

Silver electrodeposition was performed using a quasi -2D rectangular cell that consisted of two glass plates horizontally placed separated by $0.025 \mathrm{~cm}$. The cathode and the anode were made of a silver foil ( $99.999 \%$ purity), $0.025 \mathrm{~cm}$ thick. The cathode width was $1.4 \mathrm{~cm}$. The cathode-to-anode distance was $2.3 \mathrm{~cm}$. To avoid border effects and initially ensure a homogeneous primary current distribution on the cathode, a Teflon mask $0.025 \mathrm{~cm}$ thick was symmetrically placed on both sides of the cathode. The cell was mounted on a suspended table to avoid the influence of spurious mechanical vibrations on the electrochemical process. ${ }^{17,18}$

Aqueous $2.4 \times 10^{-2} \mathrm{M}$ silver sulfate $+0.5 \mathrm{M}$ sulfuric acid plating solution was prepared from Milli-Q* water and analytical reagent-grade chemicals. This working solution was used under purified nitrogen saturation. The excess of sulfuric acid behaved

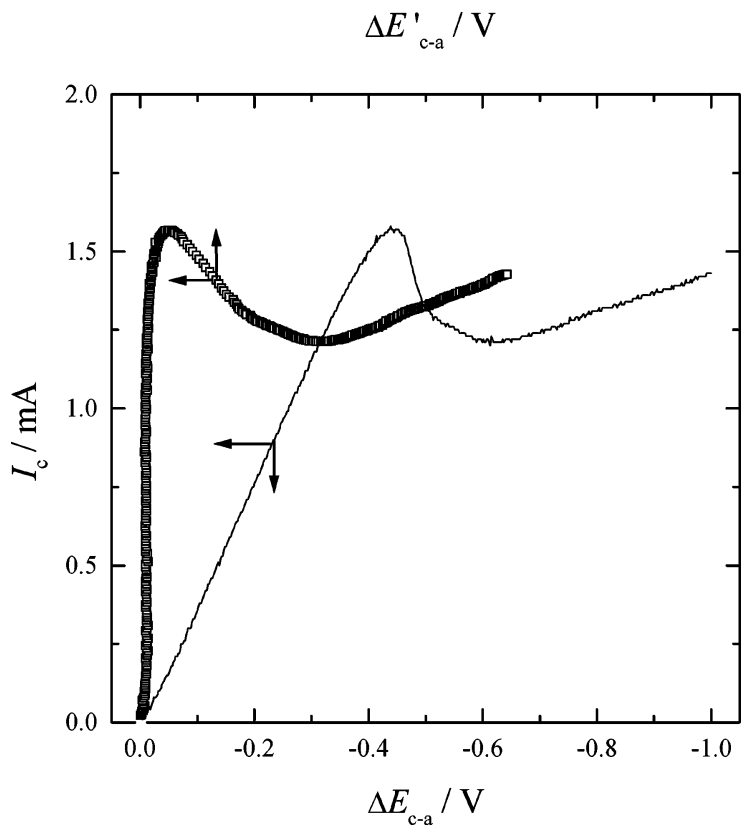

Figure 2. Cathodic polarization curves for silver electrodeposition from $0.024 \mathrm{M}$ silver sulfate $+0.5 \mathrm{M}$ aqueous sulfuric acid run at $0.1 \mathrm{~V} / \mathrm{s}$ at 298 K. Rectangular pseudo-2D rectangular cell, working electrode area $0.0375 \mathrm{~cm}^{2}$. Full trace: without ohmic drop correction. ( $\square$ ): after ohmic drop correction.

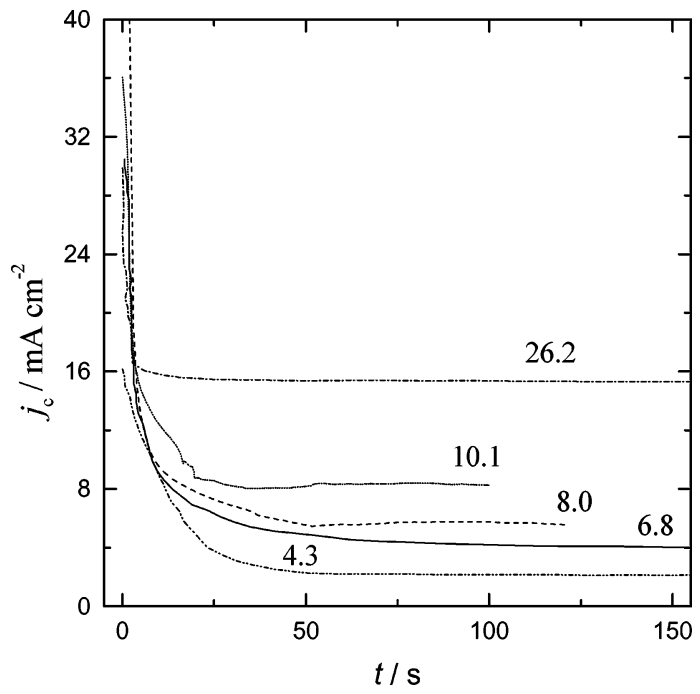

Figure 3. Experimental potentiostatic current transients for silver electrodeposition as indicated in Figure 1, at different values of $\Delta E_{\mathrm{c}-\mathrm{a}}$ and for different front displacement velocity. $\beta=4.3 \mu \mathrm{m} \mathrm{s}^{-1}, \Delta E_{\mathrm{c}-\mathrm{a}}$ $=-0.400 \mathrm{~V} ; \beta=6.8 \mu \mathrm{m} \mathrm{s}^{-1}, \Delta E_{\mathrm{c}-\mathrm{a}}=-0.430 \mathrm{~V} ; \beta=8.0 \mu \mathrm{m} \mathrm{s}^{-1}$, $\Delta E_{\mathrm{c}-\mathrm{a}}=-0.450 \mathrm{~V} ; \beta=10.1 \mu \mathrm{m} \mathrm{s}^{-1}, \Delta E_{\mathrm{c}-\mathrm{a}}=-0.520 \mathrm{~V} ; \beta=10.1$ $\mu \mathrm{m} \mathrm{s}^{-1}, \Delta E_{\mathrm{c}-\mathrm{a}}=-0.520 \mathrm{~V} ; \beta=26.2 \mu \mathrm{m} \mathrm{s}^{-1}, \Delta E_{\mathrm{c}-\mathrm{a}}=-0.650 \mathrm{~V}$. Values of $\beta$ are indicated in the plot.

as a supporting electrolyte to make the migration contribution to the diffusion controlled process smaller than $10 \%{ }^{18}$ Runs were made at $298 \mathrm{~K}$.

3.2. Interpretation and Discussion of Experimental Data. The polarization curves (Figure 2) were obtained at $0.1 \mathrm{~V} / \mathrm{s}$ to diminish the influence of the surface roughness of silver electrodeposits on the current response. They were plotted as $I_{\mathrm{c}}$ versus either $\Delta E_{\mathrm{c}-\mathrm{a}}$, the cathode-to-anode potential difference, or $\Delta E_{\mathrm{c}-\mathrm{a}}^{\prime}$, the value of $\Delta E_{\mathrm{c}-\mathrm{a}}$ after ohmic drop correction. These polarization curves exhibited well-defined cathodic limiting $\left(j_{\mathrm{c}, \mathrm{lim}}\right)$ currents from -0.25 to $-1.75 \mathrm{~V}$ that coincide with those expected for an electrochemical reaction under mass-transport 


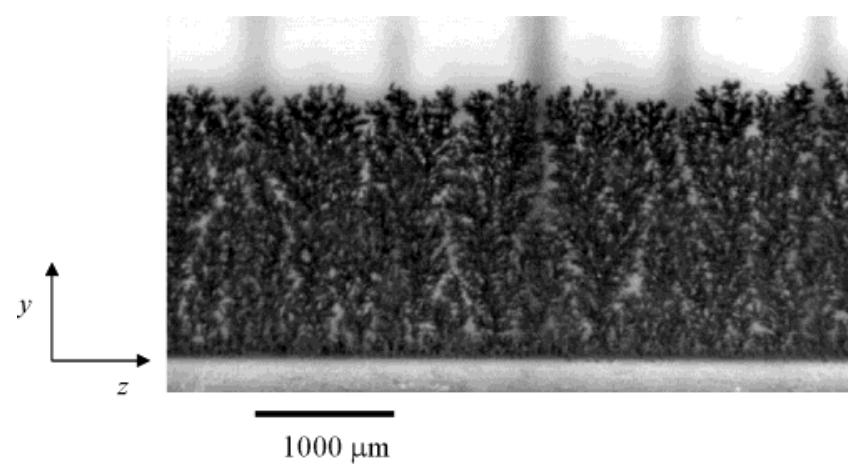

Figure 4. Photograph of a silver electrodeposit grown under limiting cathodic current on a silver plane plate using the rectangular quasi-2D cell at $298 \mathrm{~K}$. Plating solution as indicated in Figure 2. $\Delta E_{\mathrm{a}-\mathrm{c}}=-0.65$ $\mathrm{V}, t=120 \mathrm{~s}$, electrodeposition charge $=70 \mathrm{mC} / \mathrm{cm}^{2}$.

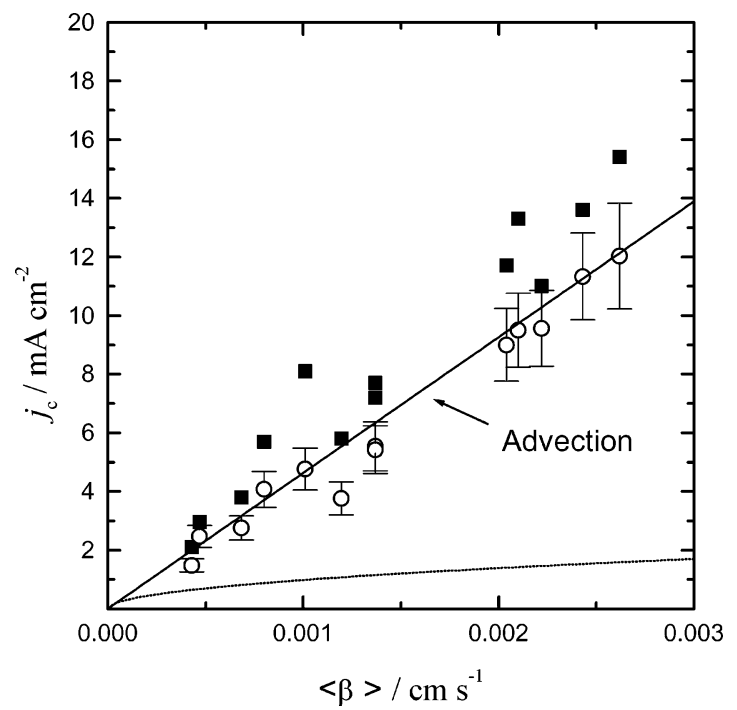

Figure 5. Plot of $j_{\mathrm{c}}$ versus $\langle\beta\rangle$ from silver electrodeposition as indicated in Figure 2 ((口) Experimental data. (O) Data after roughness factor correction). The straight line corresponds to eq 5. The lower dotted trace curve represents the $j_{\mathrm{c}}$ versus $\beta^{1 / 2}$ equation for a flow impinging perpendicularly to the plane plate electrode.

rate control. ${ }^{1,2}$ In our potentiostatic experiments, the values of $\Delta E_{\mathrm{c}-\mathrm{a}}$ were set within the potential range of the cathodic limiting current.

The experimental potentiostatic current transients (Figure 3) were run in the range $100-1200 \mathrm{~s}$, that is, in the range of charge density $0.240-13 \mathrm{C} \mathrm{cm}^{-2}$. These transients depict a complicated behavior principally due to the simultaneous contribution of the diffusion and advection, and to a lesser extent, to the roughness of quasi-2D silver electrodeposits (Figure 4). The correction for the latter was made considering the surface of the roughgrowing front accessible for diffusion. The corresponding roughness factors were evaluated from the cross-section analysis of the electrodeposit photographs considering the average diffusion layer thickness resulting from $\left(j_{\mathrm{c}, \mathrm{lim}}\right)$. Depending on the value of $\beta$, these roughness factors were between 1.2 and 1.4. These figures are consistent with the dependence of the morphology of electrodeposits on the rate of displacement of the growing front that was recently reported. ${ }^{17,18}$ After the accessible surface area correction, data fulfill reasonably well the $j_{\mathrm{c}}$ versus $\langle\beta\rangle$ plot predicted by the advection term in eq 5 (Figure 5).

Furthermore, in terms of the dimensionless eq 10 a good correlation of experimental and theoretical data was also established (Figure 6). Data scattering, in this case, can be easily

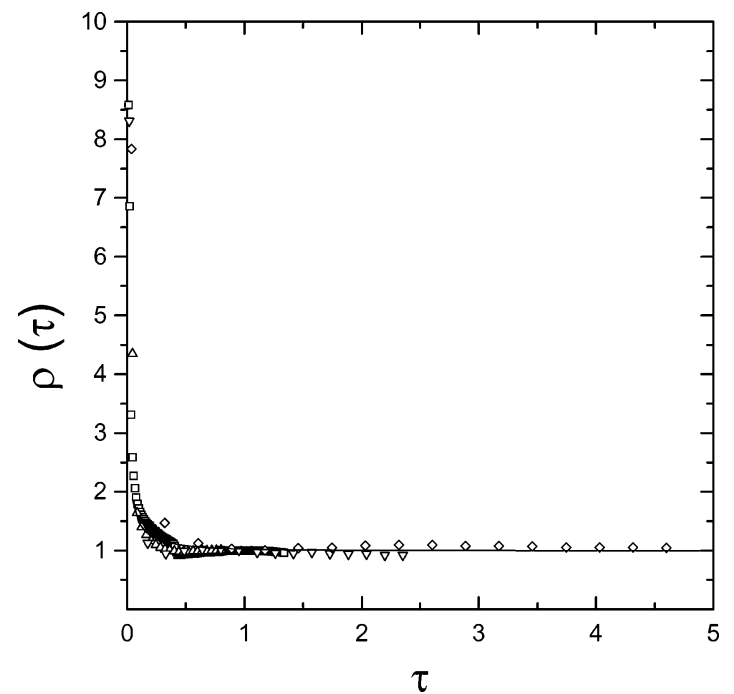

Figure 6. Dimensionless plot of $\rho(\tau)$ versus $\tau$. The full trace corresponds to eq 10 and different points represent experimental data from transients of Figure 3.

explained by the time dependence of the roughness factors that is involved in the $j_{\mathrm{c}}(\tau) /\left(j_{\mathrm{c}}\right)_{\mathrm{adv}}$ ratio. However, considering the small values of the roughness factors affecting the average diffusion layer thickness, their influence on the $j_{c}(\tau) /\left(j_{c}\right)_{\text {adv }}$ ratio tends to cancel, resulting in a surprisingly good prediction for the dimensionless correlation.

\section{Conclusions}

An analytical solution for Fick's equation for 1D solid-phase growth kinetics with a moving front whose position changes at a constant linear velocity is advanced.

The analytical solution involves a first diffusion term that includes an exponential correction factor, and a second constant advection term that depends on the front velocity.

The analytical solution also predicts a kinetic transition from diffusion dominated to advection dominated mass transfer control in going from $t \rightarrow 0$ to $t \rightarrow \infty$.

The validity of the solution was tested using potentiostatic cathodic current transient data for silver electrodeposition in a quasi-2D rectangular cell with an initially plane plate working electrode.

Experimental results from different silver electrodeposition conditions fulfill the prediction of the theory for at least about one order of magnitude in the value of $\beta$. This agreement is summarized in a dimensionless plot of data after correction for the effective area associated with diffusion.

Acknowledgment. This work was financially supported by the Consejo Nacional de Investigaciones Científicas y Técnicas (CONICET) and by the Agencia Nacional de Promoción Científica y Tecnológica of Argentina (PICT 98 No. 06-03251).

\section{References and Notes}

(1) Levich, V. E. Physicochemical Hydrodynamics; Englewood Cliffs, NJ, 1962; Chapter 2.

(2) Newman, J. S. Electrochemical Systems, 2nd ed.; Prentice-Hall: Englewood Cliffs, NJ, 1991.

(3) Moving Boundary Problems; Wilson, D. G., Solomon, A. D., Boggs, P. T., Eds.; Academic Press: New York, 1978. 589 .

(4) Tsemekhman, V.; Wettlaufer, J. S. J. Cryst. Growth 2002, 235

(5) Worster, M. G.; Wettlaufer J. S. J. Phys. Chem. B 1997, 101, 6137.

(6) Bobula, E.; Tarzia, D. A.; Twardowska, K.; Villa, L. T. SIAM Journal on Applied Mathematics 2000, 60, 1667. 
(7) Stefan, J. Zber. Akad. Wiss. Wien. 1889, 98, 473.

(8) Stefan, J. Zber. Akad. Wiss. Wien. 1889, 98, 965.

(9) Stefan, J. Ann. Phys. Chem. 1891, 47, 261.

(10) Numerical Treatment of Free Boundary Value Problem; Albrecht,

J., Collatz, L., Hoffmann, K. H., Eds.; Birkhaeuser Verlag: Basel, 1982.

(11) Free Boundary Problems: Theory and Applications; Fasano, A., Primicenio, M., Eds.; Pitman: London, 1983; Vol. 1 and 2.

(12) Crank, J. Free and Moving Boundary Problems; Clarendon Press: Oxford, 1984.

(13) Tarzia, D. Seminario sobre el Problema de Stefan y sus Aplicaciones; Institute of Mathemathics "Beppo Levi": Rosario, Argentina, 1984.

(14) Barkey, D. P.; LaPorte P. D. J. Electrochem. Soc. 1990, 137, 1656.

(15) Barkey, D. P.; Gary, P.; Ben-Jakob, E.; Miller, E.; Orr, B. J. Electrochem. Soc. 1992, 139, 1044
(16) Barkey, D. P. Advances in Electrochemical Science and Engineering; Alkire R. C., Kolb D. M., Eds.; J. Wiley-VCH: New York-Frankfurt a/M, 2002; pp 154-157.

(17) Pasquale, M. A.; Marchiano, S. L.; Arvia, A. J. Electrochim. Acto 2002, 48, 153 .

(18) Pasquale, M. A.; Marchiano, S. L.; Arvia, A. J. J. Electroanal. Chem. 2002, 532, 255

(19) Marchiano, S. L.; Arvia, A. J. Electrochim. Acta 1967, 12, 801. (20) Bazán, J. C.; Marchiano, S. L.; Arvia, A. J. Electrochim. Acta 1967, 12,821

(21) Gerischer, H.; Tischer, R. P. Z. Electrochem. 1957, 61, 1159.

(22) Budevski, W.; Bostanov, W.; Vitanov, T.; Stoinov, Z.; Kotzewa, A.; Kaischev, R. Electrochim. Acta 1966, 11, 1697.

(23) Budevski, W.; Bostanov, W.; Vitanov, T.; Stoinov, Z.; Kotzewa, A.; Kaischev, R. Phys. Status Solidi 1966, 13, 577.

(24) Porter, J. D.; Robinson, T. O. J. Phys. Chem. 1993, 97, 6696. 